Artículo Original

\title{
Redescripción de Gonolabina spectabilis (Philippi, 1863) (Dermaptera: Pygidicranidae), una tijereta de hábitat estricto en el litoral de Chile central
}

\author{
Redecription of Gonolabina spectabilis (Philippi,1863) (Dermaptera: Pygidicranidae), an \\ earwig with a strict habitat on the coastline of central Chile
}

Alejandro Vera Sánchez ${ }^{1}$

${ }^{1}$ Departamento de Biología, Universidad Metropolitana de Ciencias de la Educación, Av. José Pedro Alessandri 774, Nuñoa, Santiago de Chile. 良alejandro.vera@umce.cl

\section{ZooBank: urn:lsid:zoobank.org:pub:5A9D1CCC-DAC0-42F0-AD68-C4B1813084DF https: / / doi.org/ 10.35249/rche.47.3.21.18}

Resumen. Se entrega una redescripción de ambos sexos para Gonolabina spectabilis (Philippi, 1863), con nuevos datos sobre la morfología genital, nuevas localidades de distribución geográfica y antecedentes de su historia de vida.

Palabras clave: Gonolabininae; morfología; Polyneoptera; taxonomía.

Abstract. A redescription of both sexes is given for Gonolabina spectabilis (Philippi, 1863), with new genital characters, new geographic locations and notes on its life history.

Key words: Gonolabininae; morphology; Polyneoptera; taxonomy.

\section{Introducción}

Verhoeff (1902) crea la familia Gonolabidae para incluir los géneros Gonolabis Burr, 1900 y Gonolabina Verhoeff, 1902; este último monoespecífico con la especie Gonolabina kuhlgatzi Verhoeff, 1902. De acuerdo a este autor, Gonolabidae contendría especies con prosterno de lados convergentes hacia atrás y pigidio fusionado al tergo X. Posteriormente, Burr (1909) determina que Gonolabina es próximo al género Esphalmenus Burr, 1909, reubicando ambos géneros en la nueva subfamilia Esphalmeninae, al interior de la familia Labiduridae. Por otra parte, Rehn (1933) establece la sinonimia de Gonolabina kuhlgatzi (Verhoeff, 1902) con Forficula spectabilis Philippi, 1863. Hincks (1957), revisa la genitalia de Gonolabina y Esphalmenus concluyendo que no se relacionan, transfiere Esphalmenus y en consecuencia la subfamilia Esphalmeninae a la familia Pygidicranidae. Más tarde, Popham y Brindle (1966) crean la subfamilia Gonolabininae para acoger a Gonolabina spectabilis (Philippi, 1863) y la reubican en la familia Anisolabididae. El número de especies de Gonolabina es incrementado por Brindle (1967) al describir G. trinodosa Brindle, 1967 y G. binodosa Brindle, 1967 para los Andes de Perú. Sielfeld et al. (2018) y Hopkins et al. (2021), reconocen las tres especies de Gonolabina y su inclusión en Anisolabididae. Sin embargo, Mariani (1996) redefinió los caracteres diagnósticos de Esphelmaninae, vuelve a incluir en ella a Gonolabina, redescribe las especies y las restituye a Pygidicranidae. Pese a los arreglos

Recibido 30 Julio 2021 / Aceptado 8 Septiembre 2021 / Publicado online 30 Septiembre 2021 Editor Responsable: José Mondaca E. 
nomenclaturales y taxonómicos señalados, han sido escasos los especímenes examinados de Gonolabina spectabilis: Verhoeff (1902) indica 1 macho y 1 hembra (Tumbes, Chile) para G. kuhlgatzi; Philippi (1863) indica 1 macho y 1 hembra (Andes de la Provincia de Santiago) para F. spectabilis; Rehn (1933) estudia las descripciones anteriores y examina dos nuevos especímenes, 1 macho y 1 hembra (Valparaíso A. Faz); Brindle (1967) redescribe la especie sobre la base de otros dos especímenes, 1 macho (Valparaíso, Concón X-1884) y 1 hembra (Santiago, San Antonio); y Mariani (1996) cita 3 hembras recolectadas en los Andes peruanos (2 para Huamachuco 3.000 msnm, 21-II-1940; 1 para Canta, 800 msnm, 13-I-1954). En resumen, solo 4 machos y 7 hembras habrían sido estudiados.

El objetivo del presente estudio es redescribir la especie, sobre la base de múltiples individuos de ambos sexos, asociados mediante la observación de individuos en cópula, desde crianzas provenientes de diversas localidades de Chile, para de esta forma determinar su distribución geográfica y contribuir con antecedentes sobre su historia de vida. La redescripción que se presenta supera las limitaciones anteriores al incorporar la variabilidad morfológica y actualiza el conocimiento de la morfología genital, someramente descrita por Brindle (1967) y desconocida para la hembra.

\section{Materiales y Métodos}

Material examinado. 12 hembras y 11 machos conservados en etanol 70\% recolectados en Chile: Región de Coquimbo, Prov. Choapa, Los Vilos (bajo tronco), 3155'51"S / 71³0'50"O, III-1998, col. D. Jackson, 3ㅇ, 3ð̉. Región de Valparaíso, Prov. Valparaíso, Canelillo (bajo

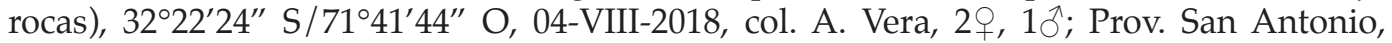

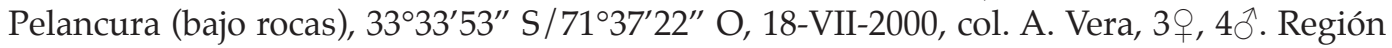
de O’Higgins, Prov. Cardenal Caro, Navidad (bajo rocas), 31 $54^{\prime} 16^{\prime \prime}$ S $/ 71^{\circ} 50^{\prime} 10^{\prime \prime}$ O, 18-II2021, col. A. Vera, 4 ㅇ, $3 \overbrace{}^{\Uparrow}$.

Especímenes secos y pinchados conservados en la colección de Entomología del Museo Nacional de Historia Natural de Chile: Región de Coquimbo, Prov. Choapa, Los Vilos, 21-X-1968, col. J. Solervicens, 1 ninfa. Región de Valparaíso, Prov. Valparaíso, Quintay (Ex.

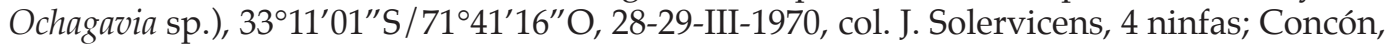

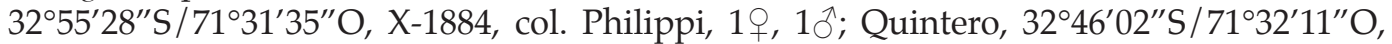

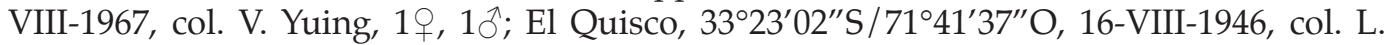

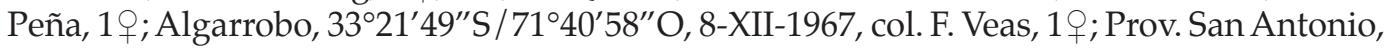
Pelancura (bajo rocas), 18-VII-2000, col. A. Vera, 3ㅇ, 10̄, 1 ninfa. Región de Los Lagos, Prov. Osorno, Osorno, 2-VII-1964, col. G. Farías, $1 \hat{\jmath}$.

Los especímenes fueron recolectados manualmente durante el día volteando piedras, troncos y plantas, fijándolos en etanol sin diluir para su conservación. Para realizar las observaciones los especímenes fueron tratados con agua destilada por 24 horas, una vez flexibilizados fueron medidos y fotografiados con un estereomicroscopio Nikon con cámara digital incorporada. Las genitalias fueron tratadas con $\mathrm{NaOH} 10 \%$ sin calentar por 24 horas, posteriormente lavadas con agua destilada y conservadas junto a los especímenes en etanol 70\%. En la morfología se sigue a Neubert et al. (2017), Hincks y Popham (1970) y Klass (2001). La clasificación sigue la propuesta de Mariani (1996).

\section{Resultados}

Gonolabina spectabilis (Philippi, 1863)

(Figs. 1a-1c, 2a-2h, 3, 4a-4i, 5a-5d, 6, 7) 


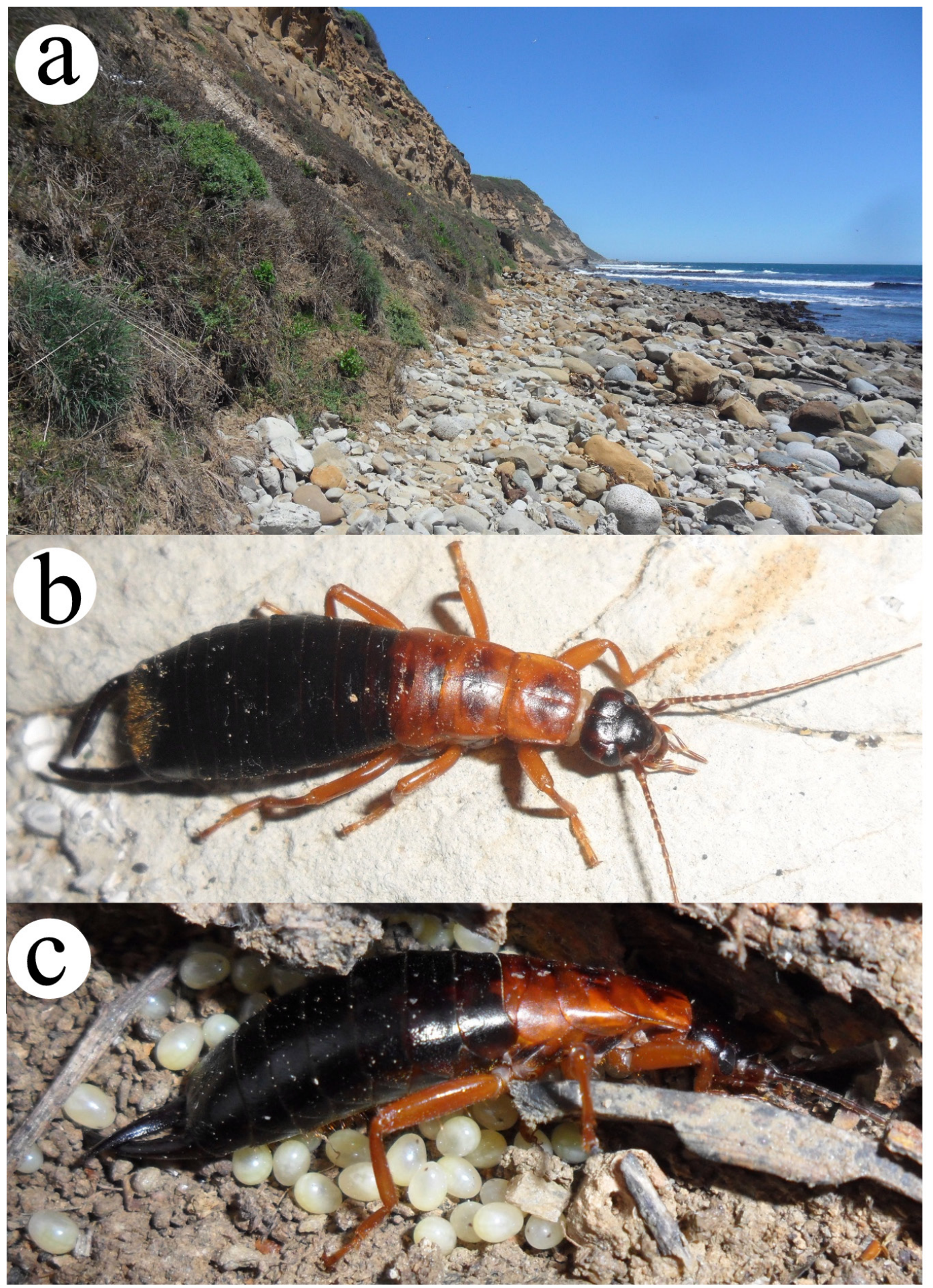

Figura 1. Gonolabina spectabilis in situ. a) Hábitat litoral entre el matorral y el intermareal (Navidad, Chile); b) Macho adulto in situ (Navidad, Chile); c) Hembra adulta in situ, con su puesta de huevos (Canelillo, Chile). / Gonolabina spectabilis in situ. a) Habitat on the coastline between matorral and intertidal (Navidad, Chile); b) Adult male in situ (Navidad, Chile); c) Adult female in situ, with her laying of eggs (Canelillo, Chile). 

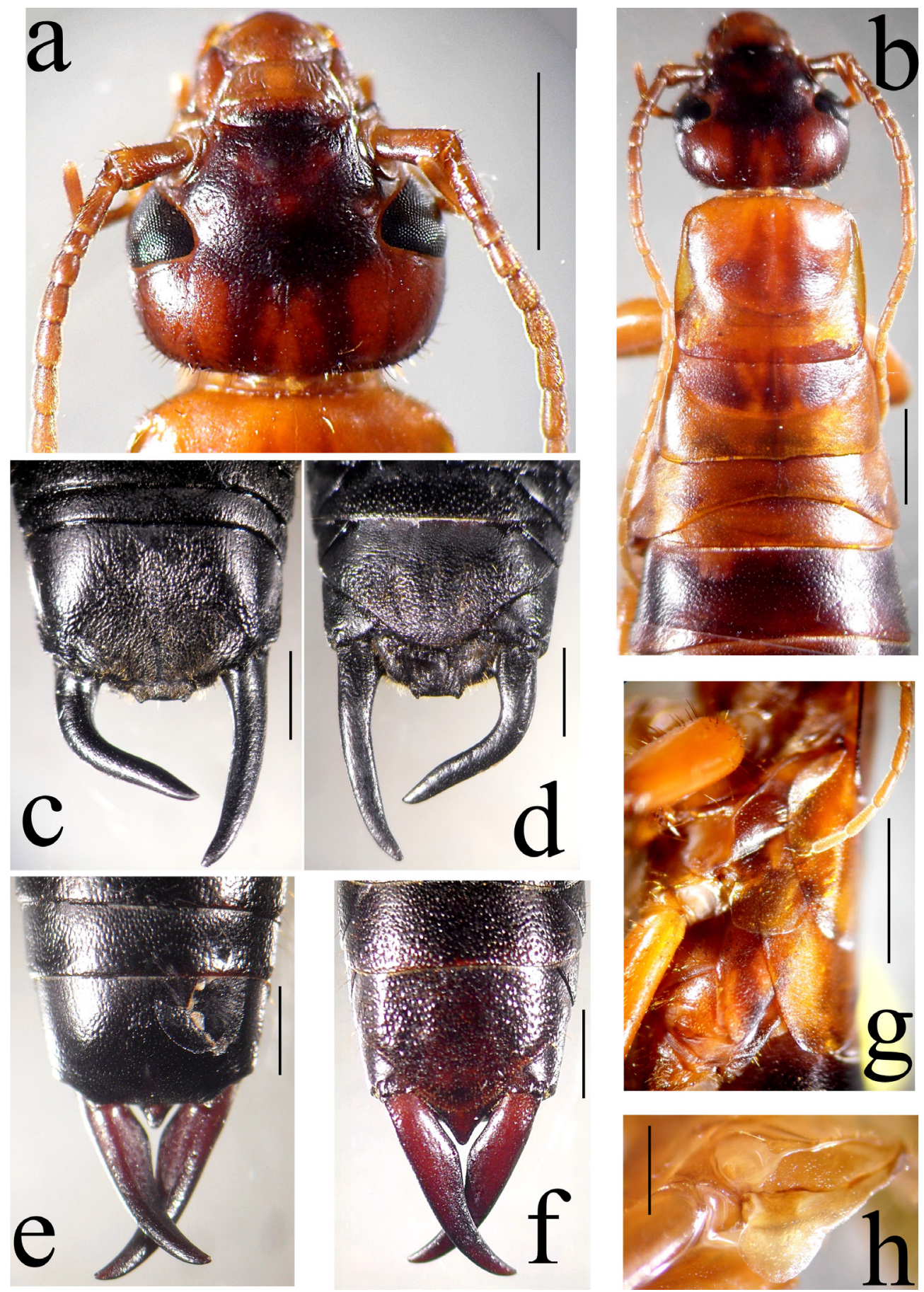

Figura 2. Morfología externa de Gonolabina spectabilis. a) Cabeza en vista dorsal, macho; b) Cabeza, tórax y tergitos I - II en vista dorsal, hembra. Extremo distal del abdomen: c) Dorsal, macho; d) Ventral, macho; e) Dorsal, hembra; f) Ventral, hembra. g) Tórax en vista lateral, hembra; h) Mesopleura en vista lateral, con expansión del epimeron, macho. Barras de escala a, b, c, d, e, f, g: 2 mm, h: $1 \mathrm{~mm}$. / External morphology of Gonolabina spectabilis. a) Head in dorsal view, male; b) Head, thorax and tergites I - II in dorsal view, female. Distal end of the abdomen: c) Dorsal, male; d) Ventral, male; e) Dorsal, female; f) Ventral, female. g) Thorax in lateral view, female; h) Mesopleure in lateral view, with expansion of the epimeron, male. Scale bars a, b, c, d, e, f, g: 2 mm, h: $1 \mathrm{~mm}$. 
Redescripción. Macho: Dimensiones en milímetros ( $\mathrm{N}=11$ ): largo corporal (sin fórceps) 1821; cabeza: ancho 3,0-3,4, largo 3,2-3,6; pronoto: largo 2,5-3,0, ancho 3,0-3,7; antena 10-13; fórceps derecho 4,3-5,0; fémur posterior 3,5-4,0; abdomen ancho máximo 5,0-5,5. Coloración (Figs. 1b, 2a): cápsula cefálica marrón oscuro a púrpura, anteclípeo, labro, labio, maxilas y palpos anaranjados, antenas anaranjadas, con el escapo oscuro y flagelo gradualmente aclarado hasta el amarillo en el extremo; tórax, patas y tergos abdominales I y II anaranjados; tergo III a X pardo oscuro, casi negro en el tergo X y los cercos (Figs. 2c, d). Cabeza (Fig. 2a): Cordiforme, ligeramente más larga que ancha, su ancho máximo se encuentra al nivel de los ojos y declina hacia posterior, vertex prominente a ambos lados de la sutura coronal, ángulos posteriores del vertex redondeados, con dos filas de setas transversales, carina parietal prominente. Cápsula cefálica de superficie con microrugosidades, mayoritariamente glabra; ojos pequeños, subtriangulares, distancia interocular 0,5 veces el ancho máximo de la cabeza; cabeza ligeramente más larga que el pronoto. Antenas con escapo dilatado distalmente, de largo similar a la suma de pedicelo y primer flagelómero, flagelo con 14 a 17 flagelómeros (solo se dispone de antenas mutiladas), primer flagelómero dos veces el largo del segundo, segundo tan largo como ancho, flagelómeros 2, 3 y 4 moniliformes, desde el quinto en adelante son claramente más largos que anchos. Tórax: Pronoto transverso, ligeramente más ancho que largo (1,2) (Fig. 1b), todos sus ángulos redondeados, anteriores con setas erizadas, márgenes rectos, laterales con un reborde; superficie plana y con microrugosidad, con surco medio longitudinal. Mesonoto transverso, dos veces más ancho que largo, 0,5 veces tan largo como el largo del pronoto, márgenes posterior y laterales rectos; superficie plana y con microrugosidad, con surco medio longitudinal; tegminas ausentes, epimeron con expansión redondeada (Figs. 2g, 2h). Metanoto transverso de margen posterior cóncavo, ángulos posteriores ampliamente redondeados, con sutura media y superficie con microrugosidad; alas ausentes. Prosterno subtriangular, de lados convergente hacia posterior, margen anterior recto, mesoesternito de margen posterior recto y ángulos posteriores redondeados, metaesternito con el margen posterior recto. Patas cortas, fémur posterior de largo similar al ancho del pronoto; razón de los tarsos: pata protorácica 3:1:4, mesotorácica 3:1:4, metatorácica 4:1:3. Abdomen gradualmente ensanchado hasta el tergo $\mathrm{V}$, luego se estrecha ligeramente hasta el tergo $X$, de tergos transversalmente convexos (Fig. 1b). Tergos I al IV de tegumento punteado, V a IX estriado longitudinalmente, último tergo abdominal transverso (Fig. 2c), de márgenes laterales rectos, área media fuertemente deprimida hacia posterior, línea media longitudinal con depresión lineal, tegumento con microrugosidad en la depresión, con setas amarillas (Figs. 1d, 2c), fuera del área deprimida escasamente punteado y sin setas amarillas. Pigidio transverso, con dos tubérculos, los tubérculos tan separados como el ancho basal del cerco. Cercos ampliamente separados en la base, cilíndricos, de ápice agudo; el derecho ligera y regularmente curvado 0,7 veces tan largo como la longitud total del cuerpo; el izquierdo fuertemente curvado en la mitad, variando desde $60^{\circ}$ a $90^{\circ}$, esta curvatura muestra amplia variabilidad (Fig. 3). Esternos de superficie punteada; placa subgenital de margen posterior redondeado a truncado (Fig. 2d), pigidio en ventral cubierto de setas amarillas. Genitalia (Figs. 4a-4i): esclerito ventral del pene simétrico, plano, ahorquillado, alargado, 1,5 veces tan largo como ancho, con sutura media completa, su cuarto distal bifurcado, parámeros agudos y subparalelos, de longitud similar a la rama ahorquillada que los soporta (Figs. 4a4f). Lóbulos distales de los penes dirigidos en oposición, lóbulo izquierdo orientado hacia adelante; lóbulo derecho orientado hacia atrás, ambos lóbulos presentan un esclerito basal (Figs. 4b, 4d, 4f); virga de longitud similar al del parámero. En protrusión el lóbulo distal del pene es de forma subcónica, de largo similar a la suma del esclerito ventral y el parámero, en su tercio proximal presenta un pequeño lóbulo digitiforme dirigido postero-ventralmente (Fig. 4d); el extremo distal esta dilatado y porta la virga, constituida por dos escleritos con forma de hacha (Fig. 4g), el esclerito de menor tamaño abraza al mayor, en su extremo dilatado su margen presenta diminutas espinas (Fig. 4h). El esclerito mayor presenta su ápice 
dilatado y extremadamente plano, su margen está rodeado por una fila de cerdas, en uno de sus extremos se abre el gonoporo (Fig. 4i).
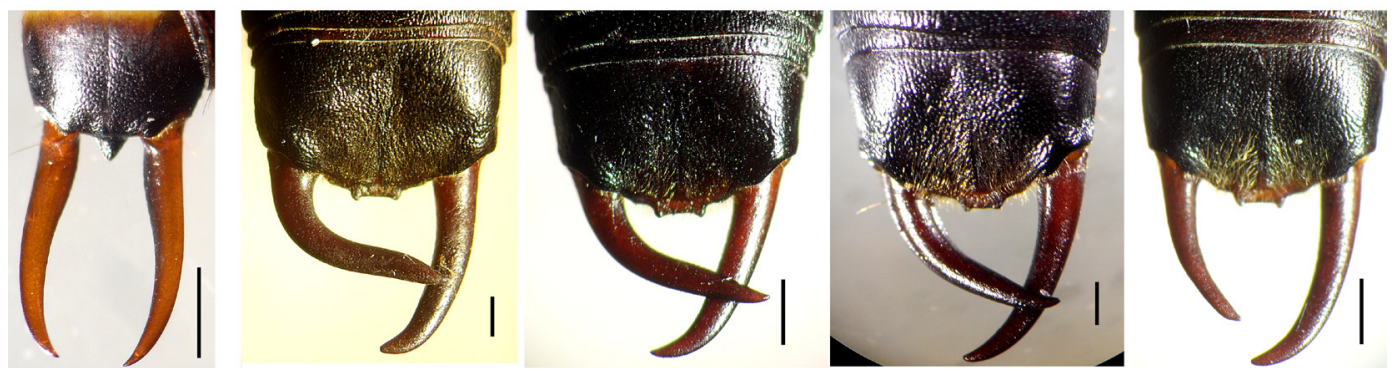

Figura 3. Variabilidad de los fórceps en machos de Gonolabina spectabilis. Localidades de izquierda a derecha: Navidad (ninfa último estadío); Pelancura; Canelillo; Navidad; Pelancura. Barra de escala: $1 \mathrm{~mm}$. / Forceps variability in Gonolabina spectabilis males. Localities from left to right: Navidad (last instar nymph); Pelancura; Canelillo; Navidad; Pelancura. Scale bar: $1 \mathrm{~mm}$.

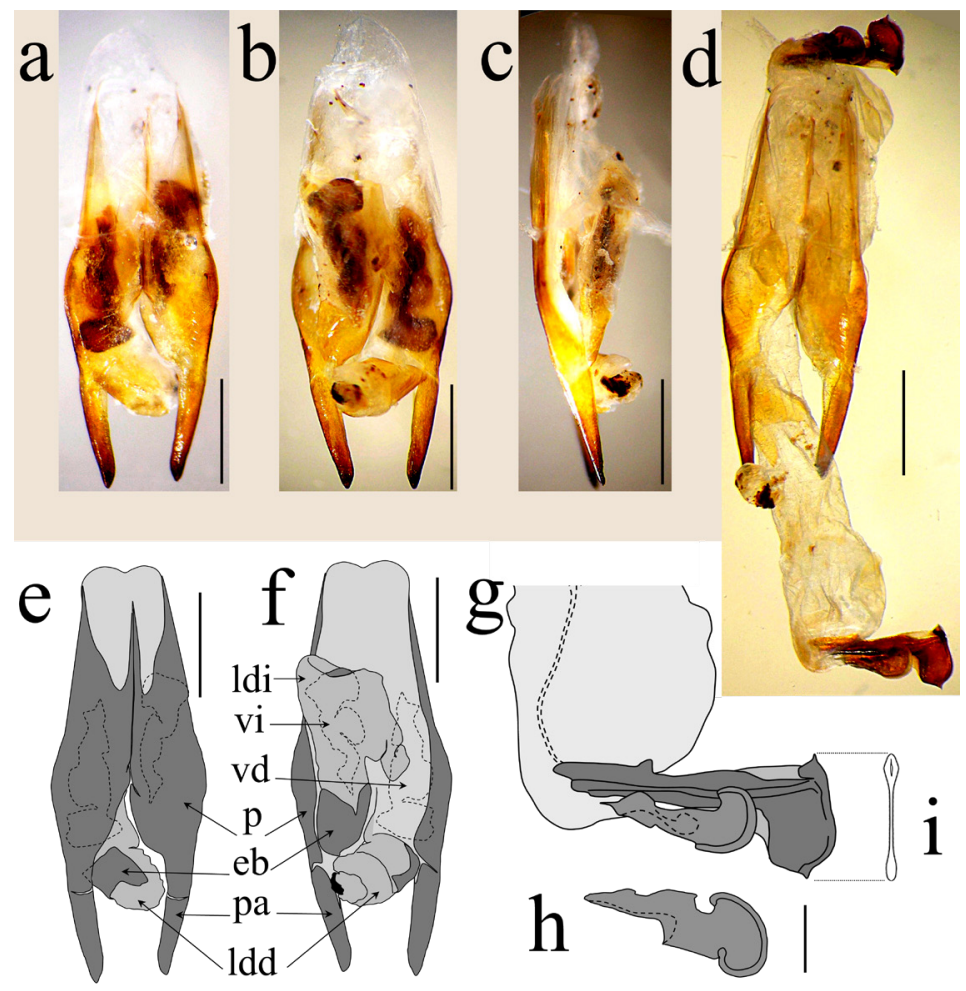

Figura 4. Genitalia masculina de Gonolabina spectabilis. Fotografías a) Vista ventral, b) Vista dorsal, c) Vista lateral, d) Ventral con los lóbulos distales de los penes en protrusión. Esquemas e) Vista dorsal, f) Vista ventral, g) Vista lateral de los ecleritos de la virga, h) Vista lateral del esclerito menor de la virga, i) Vista frontal del esclerito mayor de la virga, con el gonoporo. Abreviaturas: eb: esclerito basal del lóbulo distal; ldd: lóbulo distal del pene derecho; ldi: lóbulo distal del pene izquierdo; p: esclerito ventral del pene; pa: parámero; vi: virga izquierda; vd: virga derecha. Barras de escala a, b, c, d, e, f: 1 $\mathrm{mm} ; \mathrm{g}$, h, i; 0,5 mm. / Gonolabina spectabilis male genitalia. a) Ventral view, b) Dorsal view, c) Lateral view, d) Protruding penis distal lobule in ventral view. Line drawing: e) Dorsal view, f) Ventral view, g) Lateral view of virgae sclerites, h) Lateral view of virga minor sclerite, i) Frontal view of virga major sclerite, with the gonopore. Abbreviations: eb: basal sclerite of the distal lobe; ldd: distal lobe of the right penis; ldi: distal lobe of the left penis; p: ventral sclerite of the penis; pa: paramere; vi: left virga; vd: right virga. Scale bars a, b, c, d, e, f: $1 \mathrm{~mm}$; g, h, i: $0.5 \mathrm{~mm}$. 
Hembra: Dimensiones en milímetros ( $\mathrm{N}=12$ ): largo corporal (sin fórceps) 24,2-25,0; cabeza: ancho 3,3-3,7, largo 3,7-4,0; pronoto: largo 3,0-3,5, ancho 3,7-4,0; antena 10-15; fórceps derecho 4,5-5,5; fémur posterior 3,7-4,5; abdomen ancho máximo 5,5-6,0. Coloración (Figs. 1e, 2b) semejante al macho. Morfología semejante al macho, salvo en la forma cuadrada el pronoto (Fig. 2b) y en el abdomen de lados subparalelos, con su ancho máximo en el segmento V, tergo X trapezoidal (Fig. 2e), gradualmente angostado hacia posterior, superficie convexa, rugosa y con depresión lineal en la línea media longitudinal; pigidio cónico de ápice truncado, en vista ventral dividido en 3 regiones transversales. Cercos ligeramente curvados hacia la línea media, con el margen medial ensanchado sobre el pigidio (Figs. 2e, 2f); placa subgenital de margen posterior prominente y redondeado (Fig. 2f). Lóbulos anales con forma de hojuela, lóbulos subanales igualmente desarrollados, ambos pares presentan setas en el margen medial (Figs. 5a, 5c); gonoplaca 9 pequeña, subtriangular; gonoplaca 8 con forma de lengüeta lateral, ubicada bajo una depresión del coxosternum 8; gonapofisis 8 triangular, aguda; abertura de la espermateca con forma de una ranura estrecha. Espermateca dispuesta hacia el lado izquierdo, sobre el vestíbulo (Figs. 5a, 5c), con forma de un saco discoidal, aplanado y esclerosado, desde su margen se extienden 6 a 7 aberturas que se continúan en tubos simples de gran longitud, dispuestos enrollados sobre sí mismos y formando una caperuza sobre el margen del saco discoidal (Figs. 5b, 5d).
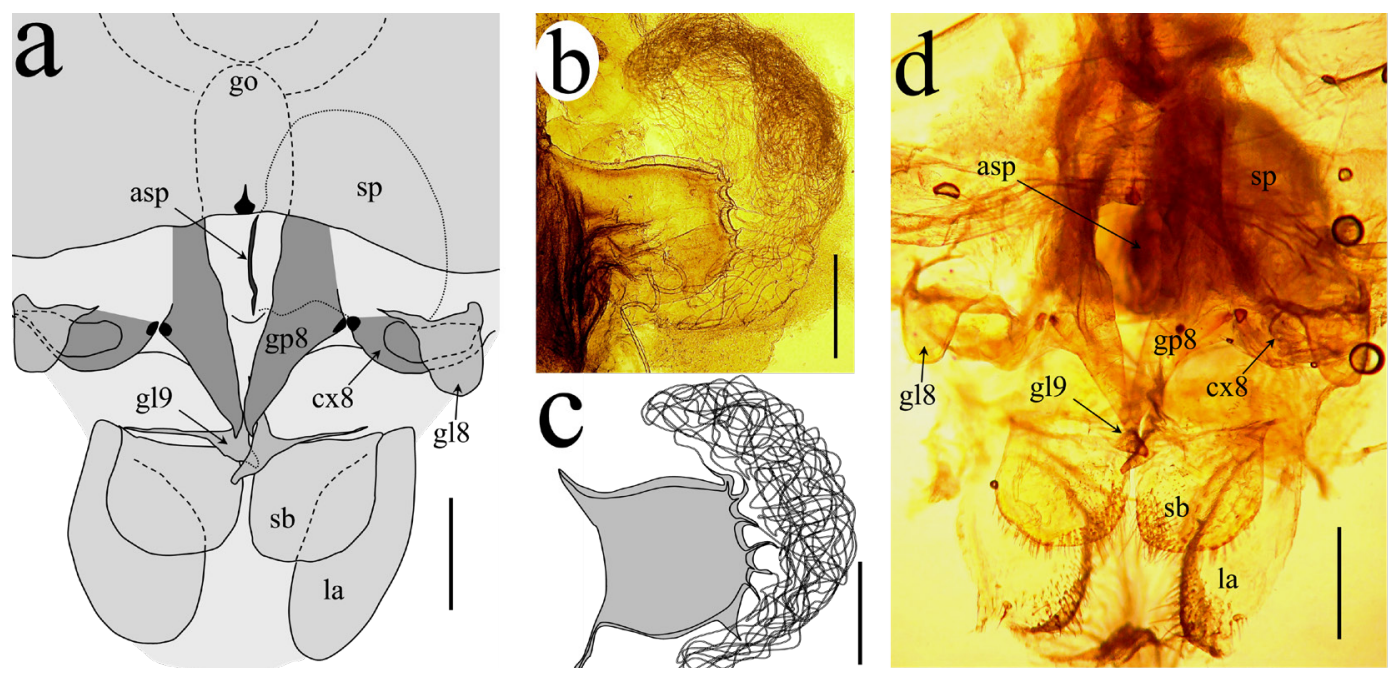

Figura 5. Genitalia femenina de Gonolabina spectabilis. a) Esquema de los escleritos asociados al vestibulum en vista ventral. b) Fotografía de La espermateca en vista lateral. c) Esquema de espermateca en vista lateral. d) Fotografía de los escleritos asociados al vestibulum en vista ventral. Abreviaturas: asp: abertura de La espermateca; cx8: coxosternum 8; g18: gonoplaca 8; g19: gonoplaca 9; gp8: gonapofisis 8; la: lóbulo anal; sb: lóbulo subanal; sp: espermateca; go: gonoporo; Barras de escala: 0,5 mm. / Gonolabina spectabilis female genitalia. a) Line drawing of the sclerites associated with the vestibulum in lateral view. b) Spermatheca in lateral view. c) Linedrawing of the spermatheca in lateral view. d) Sclerites associated with the vestibulum in ventral view. Abbreviations: asp: spermatheca opening; cx8: coxosternum 8; gl8: gonoplacs 8; g19: gonoplacs 9; gp8: gonapophysis 8; la: anal lobe; sb: subanal lobe; sp: spermatheca; go: gonopore; Scale bars: $0.5 \mathrm{~mm}$. 


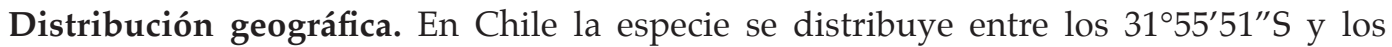

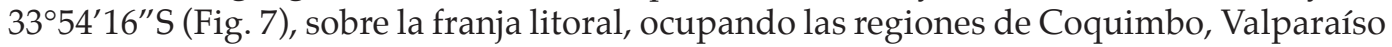
y O'Higgins. Otras localidades son indicadas con duda o descartadas (ver discusión, Fig. 7).

Historia natural. La especie se presenta en el ecotono entre el límite superior de la marea alta y sobre el margen arbustivo adyacente (Fig. 1a). Durante el día los ejemplares se encontraron preferentemente bajo rocas y troncos, adicionalmente se les ha observado entre las hojas y bajo las plantas de Ochagavia sp. (Bromeliaceae) (Solervicens comunicación personal). Solo se les ha encontrado en el litoral asociado a playas de bolones, en zonas adyacentes a acantilados con desprendimiento de rocas o con densa cubierta vegetal. Es frecuente encontrarla junto a Forficula auricularia Linnaeus, 1758, cerca del límite arbustivo y junto a anfípodos e isópodos cerca del límite intermareal.

Las hembras presentan cuidado parental, las posturas de huevos se establecen en terreno arenoso, donde estas excavan depresiones bajo las rocas (Fig. 1c). En crianza de laboratorio las ninfas no presentan dimorfismo sexual de modo que las modificaciones en los fórceps y abdomen solo se manifiestan tras la última muda (Figs. 3, 6). Entre machos y hembras vírgenes las copulas ocurren de inmediato y se repiten diariamente por alrededor de 60 días, luego ocurren de forma irregular hasta una vez por semana. En el acoplamiento la hembra siempre se encuentra con su abdomen recto, mientras que el macho se dispone posteriormente con su abdomen doblado accediendo con el lado derecho al vestíbulo genital de la hembra (Fig. 6); esto es consistente con el desarrollo del pene derecho dirigido a posterior y la espermateca acostada sobre el lado izquierdo del vestíbulo.
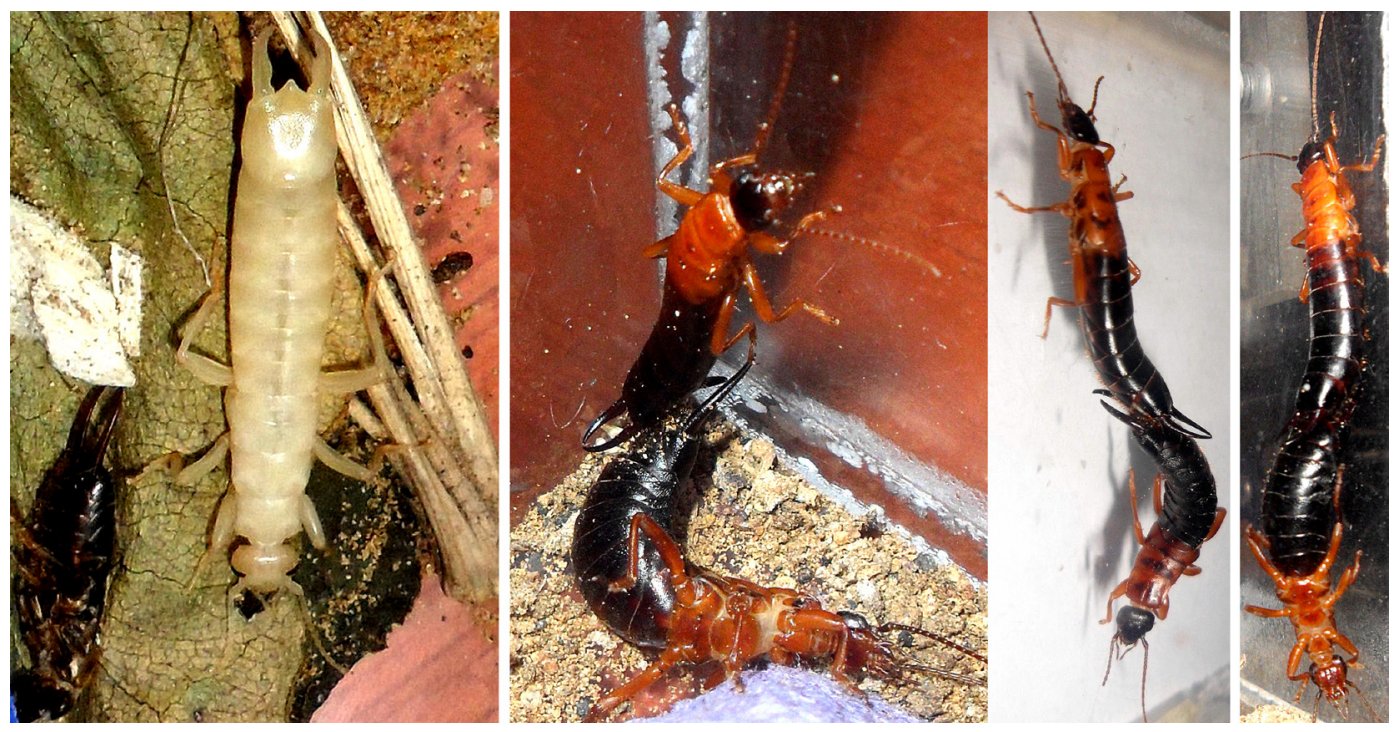

Figura 6. Gonolabina spectabilis en cautiverio. De izquierda a derecha: hembra adulta posterior a su muda; tres diferentes eventos de copulas. / Gonolabina spectabilis in captivity. From left to right: adult female after molting; three different copulation events. 


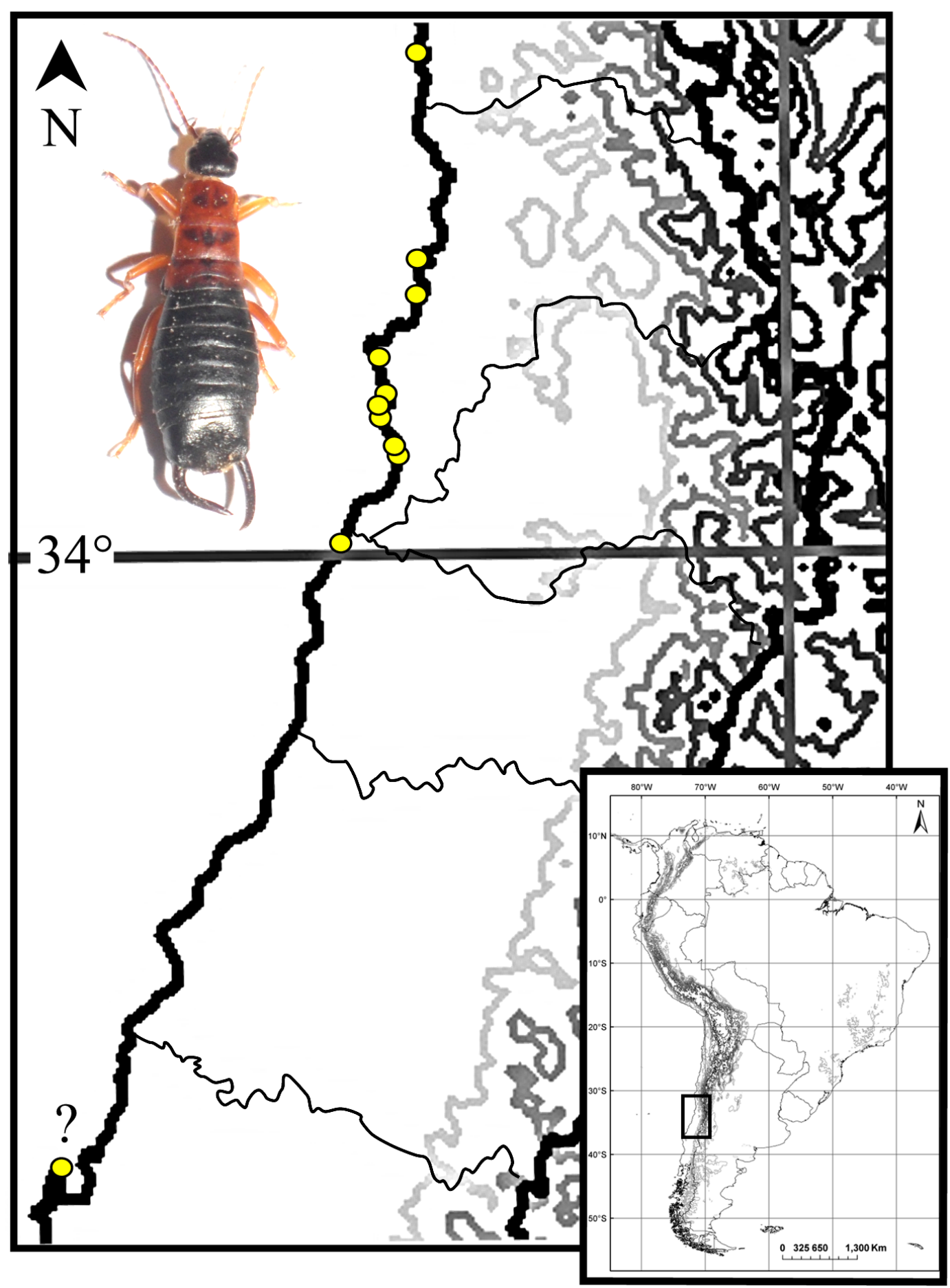

Figura 7. Distribución geográfica de Gonolabina spectabilis en Chile. Se indican las localidades citadas con puntos amarillos, los límites regionales, las líneas de nivel cada 1.000 msnm y el símbolo "?" señala la localidad de Caleta Tumbes (ver discusión). / Distribution of Gonolabina spectabilis in Chile. Shown localities with yellow dots, the regional limits, the level lines every 1000 mamsl and the "?" indicates the locality of Caleta Tumbes (see discussion). 


\section{Discusión}

Gonolabina spectabilis es la especie de mayor tamaño entre las Dermaptera nativas de Chile, su patrón de coloración corporal y la morfología del extremo abdominal permiten identificarla en ambos sexos. Sin embargo, en la redescripción de Brindle (1967) se indica la presencia de tegminias rudimentarios dispuestos lateralmente; estas estructuras no fueron identificadas, en su lugar se observó un lóbulo lateral que resultó ser una expansión no articulada del epimeron, la que se extiende sin interrupción desde la sutura pleural (Figs. 2g, 2h); Brindle (1967) indica además que los márgenes laterales y posterior del pronoto presentan un reborde levantado, esta modificación no fue observada en el margen posterior que se presenta plano. En cuanto a la genitalia masculina, la morfología del esclerito ventral del pene y parámeros es coincidente con estudios anteriores y semejante a las otras especies del género (Mariani 1996), sin embargo, la virga de G. trinodosa y G. binodosa ha sido descrita como larga y delgada, sin realizar un análisis de los lóbulos distales del pene en protrusión. En el presente estudio, la virga de G. spectabilis resulta opuesta y muy contrastante con la descripción de las otras especies congenéricas, presentando dos grandes escleritos cortos, planos y con forma de hacha. Esta diferencia en la forma de la virga que presentan las especies es coincidente con la morfología de la espermateca respectivamente; la espermateca de G. trinodosa presenta una abertura estrecha y un conducto tubular (Mariani 1994), de forma complementaria la virga se extiende en un filamento; por otra parte la espermateca de G. spectabilis presenta una abertura con forma de ranura y su primera porción es ancha, plana y esclerosada, de forma complementaria la virga se presenta como láminas planas con forma de hacha Este ajuste genital ha sido reiteradamente identificado en dermáptera, donde la virga interactúa intrínsecamente con la espermateca (Matsumura et al. 2021). En cuanto a la identificación taxonómica de las hembras, los fórceps de G. spectabilis difieren de las otras dos especies por la ausencia de un diente medial. Mariani (1996) identifica hembras provenientes de Perú sin este diente, describe los fórceps como cilíndricos con una suave curvatura en el margen interno. Esta descripción difiere de los especímenes analizados de Chile, donde los fórceps presentan una dilatación carinada y angulosa próxima al pigidio (Figs. 2f, 2g). Por otra parte, las especies G. trinodosa y G. spectabilis pueden diferenciarse fácilmente por su espermateca, la primera con forma de un saco globoso, de abertura tubular, mientras que en la segunda se presenta como un saco aplanado con múltiples tubos marginales y con abertura en forma de ranura.

En cuanto a la distribución geográfica, la especie fue citada para Perú (La Libertad y Lima) (Mariani 1996) sobre la base de hembras recolectadas a 800 y $3.000 \mathrm{msnm}$. Sin embargo, estos especímenes no corresponderían a G. spectabilis, como fue explicado anteriormente, ya que la morfología de los fórceps difiere de los especímenes chilenos, además la altitud de las localidades señaladas para Perú no se relaciona con la distribución litoral que presenta en Chile. Los registros recientes conforman un continuo de distribución en el margen litoral de Chile central, existiendo tres puntos fuera de rango: un espécimen de Osorno, distanciado de la distribución normal y fuera del litoral, lo que podría tratarse de un error de etiquetación; el material tipo de F. spectabilis, fue señalado por Philippi (1863) para la cordillera de Santiago, lo que también parece ser un error de etiquetación; Brindle (1967) indica San Antonio en Santiago, donde se confunde la localidad puerto con la capital; finalmente el material tipo de G. kuhlgatzi es citado para Tumbes, localidad que no es precisa, pudiendo tratarse de Caleta Tumbes ubicada al norte de Talcahuano en la Región del Biobío (36 $38^{\prime} 22^{\prime \prime}$ S / 7305’37" O), una localidad puerto ubicada distante al sur del límite conocido para la especie. Pese a que la distribución es consistentemente la costa central de Chile, es importante reconocer las limitaciones en el conocimiento de la diversidad de este grupo de insectos, donde las contribuciones taxonómicas, 
distribucionales, de historia de vida y las colecciones con fauna nativa son escasas, así conforme se recolecte más información podría extenderse el rango de distribución de la especie o inclusive aumentar el número de especies del género.

A partir de las restricciones del hábitat que presenta Gonolabina spectabilis, es posible desprender algunas implicancias sobre la conservación biológica de la especie, ya que se distribuye en una región con elevada intervención antrópica, donde el desarrollo inmobiliario ha aumentado por décadas en la zona, de igual forma que las faenas industriales, portuarias y el turismo intensivo. Adicionalmente la presencia de especies introducidas como Forficula auricularia que se encuentra en coexistencia con G. spectabilis. Todas estas intervenciones son amenazas no evaluadas sobre esta especie, pero que sin duda disminuyen su área de ocupación.

\section{Agradecimientos}

Se agradece a Roxana Mariani por proveer apoyo bibliográfico, a Mario Elgueta por dar acceso a la colección de entomología del Museo Nacional de Historia Natural de Chile, a Douglas Jackson por facilitar especímenes de la especie, a Susana Toledo por contribuir a incrementar el número de especímenes analizados, a Patricia Castañeda por el acceso a equipos, a Jaime Solervicens y revisores anónimos por su contribución en mejorar la redacción del manuscrito.

\section{Literatura Citada}

Brindle, A. (1967) Two new species of Gonolabina Verhoeff (Dermaptera, Carcinophoridae) from Perú. The Entomologist's Monthly Magazine, 103: 5-11.

Burr, M. (1909) Notes on the Forficularia, XV. The Esphalmeninaes. Annals and Magazine of Natural History, 8(3): 249-253.

Hincks, W.D. (1957) Dermaptera. The earwigs of South Africa. South African Animal Life, $I V:$ 33-94.

Hincks, W.D. y Popham, E.J. (1970) Dermaptera. Taxonomist's Glossary of Genitalia in Insects. (ed. Tuxen, SL.), pp. 75-80. Munksgaard, Copenhagen.

Hopkins, H., Maehr, M.D., Haas, F. y Deem, L.S. (2021) Dermaptera Species File. Version 5.0/5.0. [17-07-2021]. Disponible en: http:/ / Dermaptera.SpeciesFile.org

Klass, K.D. (2001) The female abdomen of the viviparous earwig Hemimerus vosseleri (Insecta: Dermaptera: Hemimeridae), with a discussion of the postgenital abdomen of Insecta. Zoological Journal of the Linnean Society, 131: 251-307. doi:10.1006/ zils.2001.0246

Mariani, R. (1994) Contribución al estudio anatómico de las espermatecas del orden Dermaptera (Insecta). Revista de la Sociedad Entomológica Argentina, 53(1-4): 79-82.

Mariani, R. (1996) Estudio en revisión de la subfamilia Esphalmeninae (Dermaptera, Pygidicraniidae)". Dermapterorum Catalogus XXXI. A Basic Survey for Integrated Taxonomy of the Dermaptera of the World. Pp. 9472-9567. Editor S. Sakai, Ikegamy Book Co., Tokio, Japan.

Matsumura, Y., Kamimura, Y., Lee, C-Y., Gorb, S.N. y Rajabi, H. (2021) Penetration mechanics of elongated female and male genitalia of earwigs. Scientific Reports, 11: 7920.

Neubert, D., Simon, S., Beutel, R. y Wipfler, B. (2017) The head of the earwig Forficula auricularia (Dermaptera) and its evolutionary implications. Arthropod Systematic $\mathcal{E}$ Phylogeny, 75(1): 99-124.

Philippi, R.A. (1863) Verzeichniss der im Museum von Santiago befindlichen Chilenischen Orthopteren. Zeitschrift für die Gesammten Naturwissenschaften, 21: 217-245.

Popham, E.J. y Brindle, A. (1966) Genera and species of Dermaptera. Entomologist, 99: 123$135,241-246,269-278$ 
Rehn, J. (1933) On the Dermaptera and Orthoptera of Chile. Part One. Transactions of the American Entomological Society, 59(3): 162-163.

Sielfeld, W., Jerez, V., Vera, V., González-Acuña, D., Elgueta, M., Faundez, E., Campodónico, J., Pérez, V., Gonzales, C., Moreno, L. y Taucare, A. (2018) Capítulo: Diversidad de especies 11.1 Invertebrados terrestres. En: Ministerio del medio ambiente. Biodiversidad de Chile, Patrimonios y desafíos. Tercera edición. Tomo I, 412 páginas. Santiago de Chile.

Verhoeff, K.W. (1902) Sprachüber Dermapteren II Ausfsatza: Neue ungeflügelte Eudermapteren-Gattungen, Sber Gesellschaft Naturforschender Freunde Berlin. 8. 